Journal Afrika Statistika

Vol. 5, N²12, 2010, page 288-296.

$\overline{\text { Journal Afrika Statistika }}$

\title{
Champernowne transformation in kernel quantile estimation for heavy-tailed distributions
}

\author{
Abdallah Sayah, Djabrane Yahia and Abdelhakim Necir \\ Laboratory of Applied Mathematics, Mohamed Khider University of Biskra, 07000, Algeria \\ Received 30 June 2010; Accepted 9 November 2010 \\ Copyright (C) 2010, Journal Afrika Statistika. All rights reserved
}

\begin{abstract}
By transforming a data set with a modification of the Champernowne distribution function, a kernel quantile estimator for heavy-tailed distributions is given. The asymptotic mean squared error (AMSE) of the proposed estimator and related asymptotically optimal bandwidth are evaluated. Some simulations are drawn to show the performance of the obtained results.

Résumé. En transformant un ensemble de données avec la fonction de distribution Champernowne modifiée, un estimateur à noyau du quantile pour les distributions à queues lourdes est donné. L'erreur quadratique moyenne asymptotique (AMSE) de l'estimateur proposé et la fenêtre optimale asymptotique associée sont évaluées. Des simulations sont effectuées pour montrer la performance des résultats obtenus.
\end{abstract}

Key words: Bandwidth; Champernowne distribution; Heavy tails; Kernel estimator; Quantile function. AMS 2010 Mathematics Subject Classification : 62G05, 62G32.

\section{Introduction}

The estimation of population quantiles is of great interest when a parametric form for the underlying distribution is not available. It plays an important role in both statistical and probabilistic applications, namely: the goodness-of-fit, the computation of extreme quantiles and Value-at-Risk in insurance business and financial risk management. Also, a large class of actuarial risk measures can be defined as functionals of quantiles (see, Denuit et al. [8]).

Quantile estimation has been intensively used in many fields, see Azzalini [1], Harrel and Davis [11], Sheather and Marron [19], Ralescu and Sun [16], Chen and Tang [7]. Most of the existing estimators suffer from either a bias or an inefficiency for high probability levels. To solve this inconvenience, we suggest to use the so-called transformed kernel estimate, firstly used in the density estimation context, by Devroye and Györfi [9] for heavy-tailed observations. The idea is to transform the initial observations $\left\{X_{1}, \ldots, X_{n}\right\}$ into a sample $\left\{Z_{1}, \ldots, Z_{n}\right\}:=\left\{T\left(X_{1}\right), \ldots, T\left(X_{n}\right)\right\}$, where $T$ is a given function having values in $(0,1)$. Buch-Larsen et al. [2] suggested to choose $T$ so that $T(X)$ is close to the uniform distribution. They proposed a kernel density estimation of heavy-tailed distributions based on a transformation of the original data set with a modification of the Champernowne cumulative distribution function (cdf) (see, Champernowne $[4,5]$ ). While Bolancé et al. [3] proposed the Champernowne-inverse beta transformation in kernel density estimation to model insurance claims and showed that their method is preferable to other transformation density estimation approaches for distributions that are Pareto-like.

Recently, in order to correct the bias problems, Charpentier and Oulidi [6] suggested several nonparametric quantile estimators based on the beta-kernel and applied them to transformed data. For nonparametric estimation, the bandwidth controls the balance between two considerations: bias and variance. Furthermore, the mean squared error (MSE) which is the sum of squared bias and variance, provides a composite measure of performance. Therefore, optimality

Abdallah Sayah: sayahabdallah@yahoo.fr

Djabrane Yahia: yahia_dj@yahoo.fr

Abdelhakim Necir: ah.necir@univ-biskra.dz 
A. Sayeh, D. Yahia and A. Necir, Journal Afrika Statistika, Vol. 5, Nº12, 2010, page 288-296.

Champernowne transformation in kernel quantile estimation for heavy-tailed distributions

in the sense of MSE is not seriously swayed by the choice of the kernel but is affected by that of the bandwidth (for more details, see Wand and Jones [21]. In this paper, we propose a new estimator of the quantile function, based on the modified Champernowne transformation and we obtain an expression for the value of the smoothing parameter that minimizes the AMSE of the obtained estimator. The use of this transformation in kernel estimation of quantile functions for heavy-tailed distributions improves the already existing results.

The rest of the paper is organized as follows. In Section 2, the kernel quantile estimation is given. Section 3 is devoted to the Champernowne transformation and the estimation procedure. In Section 4, we propose an asymptotically optimal bandwidth selection. A simulation study is carried out in Section 5. Finally we outline some concluding remarks in Section 6 .

\section{Kernel quantile estimation}

Let $X_{1}, X_{2}, \ldots$, be independent and identically distributed (iid) random variables (rv's) drawn from an absolutely continuous (cdf) $F$ with probability density function (pdf) $f$. For each interger $n$, let $X_{1, n} \leq \ldots \leq X_{n, n}$ denote the order statistics pertaining to the sample $X_{1}, \ldots, X_{n}$. We define the $p t h$ quantile $Q_{X}(p)$ as the left-continuous inverse of $F$ as

$$
Q_{X}(p):=\inf \{x \in \mathbb{R}: F(x) \geq p\}, \quad 0<p<1 .
$$

A basic estimator of $Q_{X}(p)$, is the sample quantile $Q_{n}(p)=X_{[n p]+1, n}$ where $[x]$ denotes the integer part of $x \in \mathbb{R}$. Suppose that $K$ is a pdf symmetric about 0 and $h:=h_{n}$ is a sequence of real numbers (called bandwidth) such that $h \rightarrow 0$ as $n \rightarrow \infty$. The classical kernel quantile estimator (CKQE) was introduced by Parzen [15] in the following form:

$$
\tilde{Q}_{n, X}(p):=\sum_{i=1}^{n} X_{i, n} \int_{\frac{i-1}{n}}^{\frac{i}{n}} K_{h}(x-p) d x,
$$

where $K_{h}(t):=K(t / h) / h$. Yang [22] established the asymptotic normality and the mean squared consistency of $\tilde{Q}_{n, X}(p)$, while Falk ([10] showed that the asymptotic performance of $\tilde{Q}_{n, X}(p)$ is better than that of the empirical sample quantile. Sheather and Marron [19] gave the AMSE of $\tilde{Q}_{n, X}(p)$. For further details on kernel-based estimation, see Silverman [20] and Wand and Jones [21].

\section{Champernowne transformation and estimation procedure}

In the context of quantile estimation, if $T$ is strictly increasing, the $p^{\text {th }}$ quantile of $T(X)$ is equal to $T\left(Q_{X}(p)\right)$. Firstly, we use a parametric transformation $T$, namely the modified Champernowne cdf as proposed by Buch-Larsen et al. [2] when fitting insurance claims:

$$
T_{\alpha, M, c}(x):=\frac{(x+c)^{\alpha}-c^{\alpha}}{(x+c)^{\alpha}+(M+c)^{\alpha}-2 c^{\alpha}}, \quad x \geq 0,
$$

with parameters $\alpha>0, M>0$ and $c \geq 0$. The associated pdf is

$$
t_{\alpha, M, c}(x):=\frac{\alpha(x+c)^{\alpha-1}\left((M+c)^{\alpha}-c^{\alpha}\right)}{\left((x+c)^{\alpha}+(M+c)^{\alpha}-2 c^{\alpha}\right)^{2}}, \quad x \geq 0 .
$$

This distribution is of Pareto type, that is

$$
t_{\alpha, M, c}(x) \sim \frac{\alpha\left((M+c)^{\alpha}-c^{\alpha}\right)}{x^{\alpha+1}}, \quad \text { as } x \rightarrow \infty .
$$

The idea is to transform the initial data $\left\{X_{1}, \ldots, X_{n}\right\}$ into $\left\{Z_{1}, \ldots, Z_{n}\right\}$, where $Z_{i}:=T\left(X_{i}\right), i=1, \ldots, n$. This can be assumed to have been produced by a $(0,1)$-uniform rv $Z$. Thus, (1) yields the transformed kernel quantile estimator (TKQE)

where $T^{-1}$ is the inverse of $T$ and

$$
\hat{Q}_{n, X}(p):=T^{-1}\left(\hat{Q}_{n, Z}(p)\right)
$$

$$
\hat{Q}_{n, Z}(p):=\sum_{i=1}^{n} Z_{i, n} \int_{\frac{i-1}{n}}^{\frac{i}{n}} K_{h}(z-p) d z .
$$

The estimation procedure is described as follows: 
1. Compute the estimates $(\hat{\alpha}, \hat{M}, \hat{c})$ of the parameters of the modified Champernowne distribution (2). Notice that $T_{\alpha, M, 0}(M)=0.5$, this suggests that $M$ can be estimated by the empirical median (see Lehmann [14]). Then, estimate the pair $(\alpha, c)$ which maximizes the log-likelihood function (see, Buch-Larsen et al. [2]):

$$
\begin{aligned}
l(\alpha, c) & =n \log \alpha+n \log \left((M+c)^{\alpha}-c^{\alpha}\right)+(\alpha-1) \sum_{i=1}^{n} \log \left(X_{i}+c\right) \\
& -2 \sum_{i=1}^{n} \log \left(\left(X_{i}+c\right)^{\alpha}+(M+c)^{\alpha}-2 c^{\alpha}\right) .
\end{aligned}
$$

2. Transform the data $X_{1}, \ldots, X_{n}$ into $Z_{1}, \ldots, Z_{n}$ by

$$
Z_{i}=T_{\hat{\alpha}, \hat{M}, \hat{c}}\left(X_{i}\right), \quad i=1, \ldots, n .
$$

The resulting transformed data belong to the interval $(0,1)$.

3. Using (3), calculate the kernel quantile estimator $\hat{Q}_{n, Z}(p)$ of the transformed data: $Z_{1}, \ldots, Z_{n}$.

4. The resulting TKQE of the original data $X_{1}, \ldots, X_{n}$ is given by

$$
\hat{Q}_{n, X}(p)=T_{\hat{\alpha}, \hat{M}, \hat{c}}^{-1}\left(\hat{Q}_{n, Z}(p)\right)
$$

\section{Asymptotic theory and bandwidth selection}

Let $X_{1}, \ldots, X_{n}$ be iid rv's with cdf $F$ and pdf $f$. For each $p$ in $(0,1)$, let $\hat{Q}_{n, X}(p)$ be the TKQE $(5)$ of $Q_{X}(p)$.

Theorem 1. Assume that $Q_{Z}(\cdot)$ is two-times diffirentiable in a nieghborhood of $p \in(0,1)$ with continuous second derivative. Assume further that the kernel $K$ has compact support and fulfills:

$$
\int K(t) d t=1, \quad \int t K(t) d t=0 \quad \text { and } \quad \int t^{2} K(t) d t<\infty .
$$

Then the bias and the variance of $\hat{Q}_{n, X}(p)$ are respectively

$$
\operatorname{Bias}\left(\hat{Q}_{n, X}(p)\right)=\frac{h^{2}}{2}\left[\left(T^{-1}\right)^{\prime \prime}\left(Q_{Z}(p)\right) Q_{Z}^{\prime 2}(p)+\left(T^{-1}\right)^{\prime}\left(Q_{Z}(p)\right) Q_{Z}^{\prime \prime}(p)\right] \mu_{2}(K)+o\left(h^{2}\right),
$$

and

$$
\operatorname{Var}\left(\hat{Q}_{n, X}(p)\right)=\left(\left(T^{-1}\right)^{\prime}\left(Q_{Z}(p)\right) Q_{Z}^{\prime}(p)\right)^{2}\left(\frac{p(1-p)}{n}-\frac{h}{n} \varphi(K)\right)+o\left(\frac{h}{n}\right)
$$

where $\mu_{2}(K):=\int t^{2} K(t) d t, \varphi(K):=2 \int t K(t)\left(\int_{-\infty}^{t} K(s) d s\right) d t, Q_{Z}^{\prime}$ and $Q_{Z}^{\prime \prime}$ are the first and the second derivatives of $Q_{Z}$. The value of $h$ that minimizes the AMSE of $\hat{Q}_{n, X}(p)$ is

$$
h_{o p t, X}:=\left(\frac{\left(\left(T^{-1}\right)^{\prime}\left(Q_{Z}(p)\right) Q_{Z}^{\prime}(p)\right)^{2} \varphi(K)}{n \Psi_{T, Q}^{2}(p) \mu_{2}^{2}(K)}\right)^{1 / 3}
$$

where

$$
\Psi_{T, Q}(p):=\left(T^{-1}\right)^{\prime \prime}\left(Q_{Z}(p)\right) Q_{Z}^{\prime 2}(p)+\left(T^{-1}\right)^{\prime}\left(Q_{Z}(p)\right) Q_{Z}^{\prime \prime}(p)
$$

The associated AMSE is

$$
\begin{aligned}
A M S E_{h_{o p t, X}} & :=n^{-1}\left\{p(1-p)\left(\left(T^{-1}\right)^{\prime}\left(Q_{Z}(p)\right) Q_{Z}^{\prime}(p)\right)^{2}\right. \\
& \left.-\frac{3}{4}\left(\left(\left(T^{-1}\right)^{\prime}\left(Q_{Z}(p)\right) Q_{Z}^{\prime}(p)\right)^{8} \varphi^{4}(K)\left(n \Psi_{T, Q}^{2}(p) \mu_{2}^{2}(k)\right)^{-1}\right)^{1 / 3}\right\} .
\end{aligned}
$$


Proof. The proof is the same as for the classical kernel quantile estimator, (see Falk [10] and Sheater and Marron [19]). It suffices to replace $Q_{X}(p)$ by $T^{-1}\left(Q_{Z}(p)\right)$. Suppose that $Z$ has pdf $g$ and cdf $G$. In the cases where $g$ is not symmetric or symmetric with $p \neq 0.5$, Sheater and Marron [19] gave the $A M S E$ of $\hat{Q}_{n, Z}(p)$ :

$$
A M S E\left(\hat{Q}_{n, Z}(p)\right)=\frac{p(1-p)}{n} Q_{Z}^{\prime 2}(p)+\frac{1}{4} h^{4} Q_{Z}^{\prime \prime 2}(p) \mu_{2}^{2}(K)-\frac{h}{n} Q_{Z}^{\prime 2}(p) \varphi(K) .
$$

If $Q_{Z}^{\prime}(p)>0$, the asymptotically optimal bandwidth for $\hat{Q}_{n, Z}(p)$ is

$$
h_{o p t, Z}=\left(\frac{Q_{Z}^{\prime 2}(p) \varphi(K)}{n Q_{Z}^{\prime \prime 2}(p)^{2} \mu_{2}(K)^{2}}\right)^{1 / 3} .
$$

When $g$ is symmetric and $p=0.5$, we have

$$
A M S E\left(\hat{Q}_{n, Z}(0.5)\right)=\frac{1}{n} Q_{Z}^{\prime 2}(0.5)\left\{0.25-0.5 h \varphi(K)+\frac{1}{n h} \int K^{2}(t) d t\right\} .
$$

Remark 1. If $Q_{X}^{\prime}(p)>0$, the asymptotically optimal bandwidth for the CKQE $\tilde{Q}_{n, X}(p)$ is

$$
h_{o p t, C}=\left(\frac{Q_{X}^{\prime 2}(p) \varphi(K)}{n Q_{X}^{\prime \prime 2}(p)^{2} \mu_{2}(K)^{2}}\right)^{1 / 3}
$$

Remark 2. The first and the second derivatives of $Q_{Z}$ are

$$
Q_{Z}^{\prime}(p)=\frac{1}{g\left(Q_{Z}(p)\right)}=\frac{T^{\prime}\left(Q_{X}(p)\right)}{f\left(Q_{X}(p)\right)}
$$

and

$$
\begin{aligned}
Q_{Z}^{\prime \prime}(p) & =\frac{-g^{\prime}\left(Q_{Z}(p)\right)}{g^{3}\left(Q_{Z}(p)\right)} \\
& =-\frac{f^{\prime}\left(Q_{X}(p)\right) T^{\prime}\left(Q_{X}(p)\right)-f\left(Q_{X}(p)\right) T^{\prime \prime}\left(Q_{X}(p)\right)}{f^{3}\left(Q_{X}(p)\right)}
\end{aligned}
$$

\section{Simulation study}

The main purpose of this section is to compare the CKQE $\tilde{Q}_{n, X}(p)$ and the TKQE $\hat{Q}_{n, X}(p)$. The distributions used in simulation are described in Table 1.

Table 1. Distributions used in the simulation study

\begin{tabular}{lll}
\hline Distribution & Density for $x>0$ & Parameters \\
\hline Burr $(\alpha, \gamma, \theta)$ & $\frac{\alpha \gamma(x / \theta)^{\gamma}}{x\left(1+(x / \theta)^{\gamma}\right)^{\alpha+1}}$ & $(\alpha, \gamma, \theta)=(2,3,1)$ \\
Paralogistic $(\alpha, \theta)$ & $\frac{\alpha^{2}(x / \theta)^{\alpha}}{x\left(1+(x / \theta)^{\alpha}\right)^{\alpha+1}}$ & $(\alpha, \theta)=(3, .5)$ \\
Mixture of $\rho \log -\operatorname{normal}(\mu, \sigma)$ & $\rho \frac{1}{\sqrt{2 \pi \sigma^{2}} x} \exp \left\{-\frac{(\log x-\mu)^{2}}{2 \sigma^{2}}\right\}$ & $(\rho, \mu, \sigma, \alpha, \theta)=(0.7,0,1,1,1)$ \\
and $(1-\rho) \operatorname{Pareto}(\alpha, \theta)$ & $+(1-\rho) \frac{\alpha(x / \theta)}{x(1+(x / \theta))^{\alpha+1}}$ & \\
\hline
\end{tabular}

Note that, the mixture of log-normal and Pareto distributions was previously used in Buch-Larsen et al. [2] and Charpentier and Oulidi [6]. The performance of the estimators is measured by the AMSE criteria:

$$
A M S E:=\frac{1}{N} \sum_{s=1}^{N}\left(\hat{Q}_{n, X}^{(s)}(p)-Q(p)\right)^{2}
$$


where $\hat{Q}_{n, X}^{(s)}(p)$ is the quantile correponding to the $s^{t h}$ simulated sample $\left\{X_{1}^{(s)}, \ldots, X_{n}^{(s)}\right\}$ and $N$ is the number of replications. The algorithm used to estimate the quantile function with level $p \in(0,1)$ is described as follows:

1. Generate a sample $X_{1}, \ldots, X_{n}$ of size $n$.

2. Estimate $M$ by the empirical median $\hat{M}$, solution of $T_{\alpha, M, 0}(M)=0.5$.

3. Estimate the pair $(\alpha, c)$ maximizing the log-likelihood function (4).

4. Transform $X_{1}, \ldots, X_{n}$ into $Z_{1}, \ldots, Z_{n}$ :

$$
Z_{i}=T_{\hat{\alpha}, \hat{M}, \hat{c}}\left(X_{i}\right), \quad i=1, \ldots, n
$$

5. Compute the estimate $\hat{Q}_{n, Z}(p)$ by choosing the Epanechnikov kernel: $K(t)=\frac{3}{4}\left(1-t^{2}\right) \mathbf{1}_{(|t|<1)}$.

6 . The resulting TKQE of the original data is

$$
\hat{Q}_{n, X}(p)=T_{\hat{\alpha}, \hat{M}, \hat{c}}^{-1}\left(\hat{Q}_{n, Z}(p)\right) .
$$

7. The CKQE is directly obtained from the original data, where the bandwidth $h:=h_{o p t, C}$ is such as in (8).

We draw from the four distributions samples of size 50, 100, 500 and compute the TKQE and CKQE for different values of $p$ in $(0,1)$. In Figures 1-4, the solid (black), dashed (red) and dotted (blue) lines, respectively, represent the true quantile $Q(p)$, the CKQE and the TKQE. On these figures, we observe that our TKQE is always better than the CKQE, especially when $p$ is close to 1 .

Secondly, we fix the sample size at 200 and compute both the TKQE and CKQE for probability levels $p \in$ $\{.05, .10, .25, .50, .75, .90, .95\}$. We repeat the process $N=200$ times and we take the average. The results are summarized in Tables $2-5$ where we see that the TKQE is better than the CKQE for high probability levels $p \in\{.75, .90, .95\}$. Table 4 is based on the mixture $30 \%$ log-normal and $70 \%$ Pareto distributions. Both estimators are equal for $p \in\{.05, .10, .25, .50\}$.

Next, we sample, 200 times, from the four distributions sets of sizes 50, 100, 500 and compute the TKQE and CKQE with their $A M S E^{\prime} s$ for levels $p \in\{.75, .90, .95\}$. The respective results are given in Tables 6,7 and 8 . It is clear that, for large probability levels, the transformation-based approach gives results of higher quality with respect to the classical procedure. Note that, under the classical estimation, some $A M S E^{\prime} s$ are seriously bad when samples come from mixture distributions, especially when $70 \%$ of Pareto distribution is considered. The same remark is observed in Charpentier and Oulidi [6] (see their table's 13-18 pages 52-53).
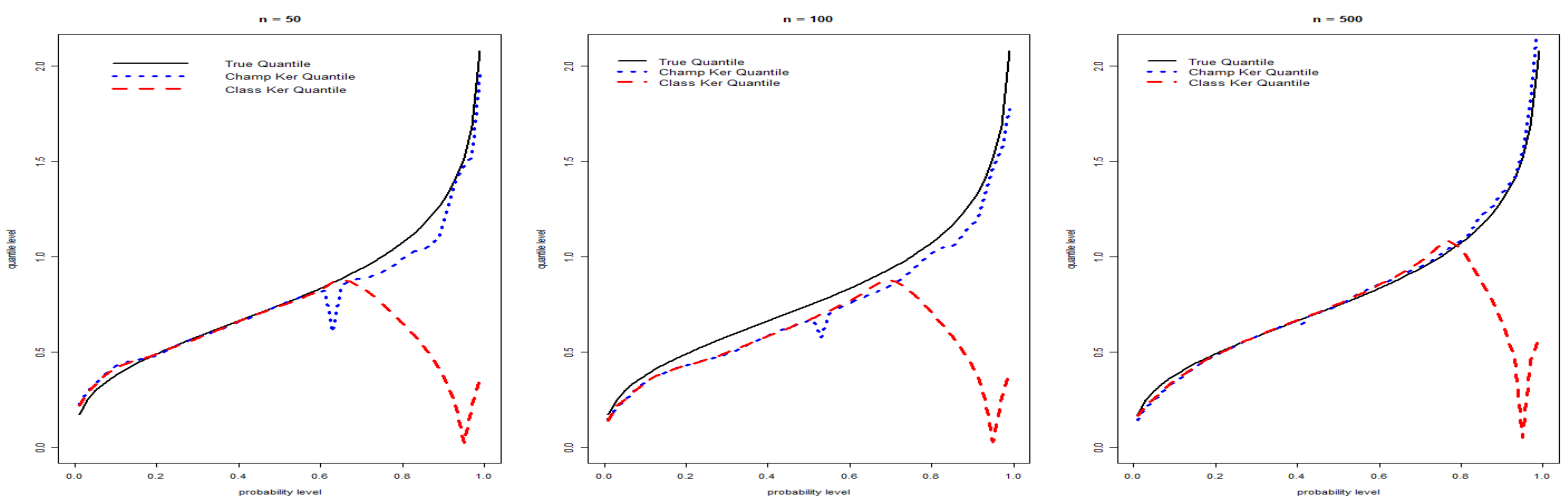

Figure 1: True quantile, classical and transformed $p^{\text {th }}$ quantile estimators : Burr distribution, $n=50$, 100 and 500, $p \in(0,1)$. 

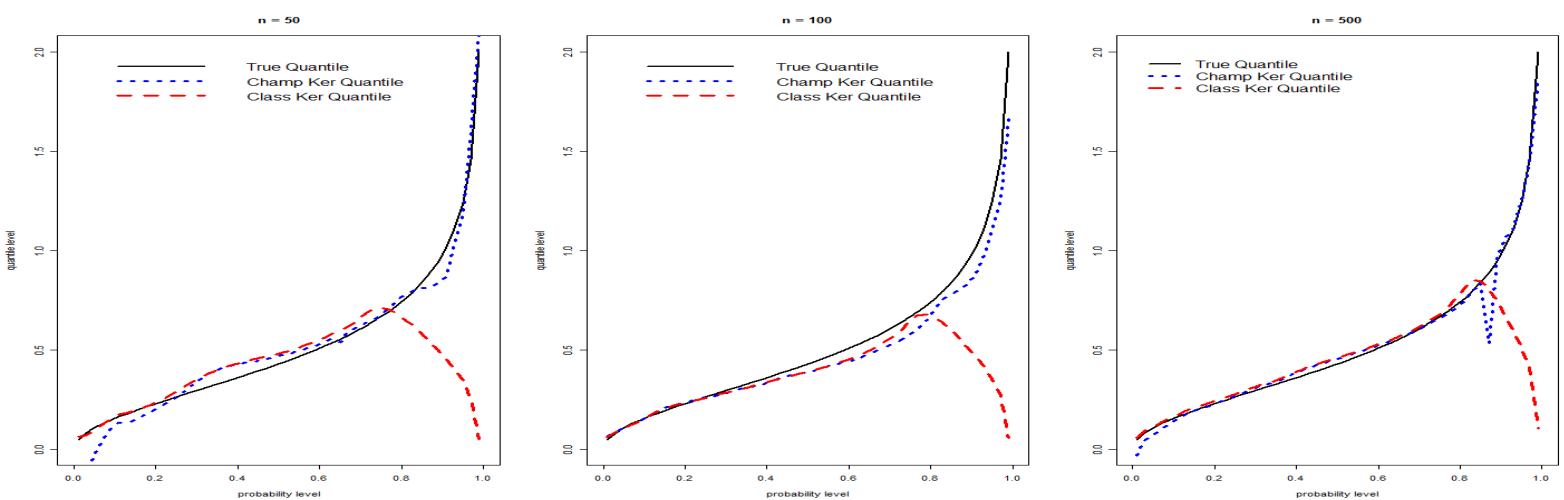

Figure 2: True quantile, classical and transformed $p^{\text {th }}$ quantile estimators : Paralogistic distribution, $n=50,100$ and $500, p \in(0,1)$
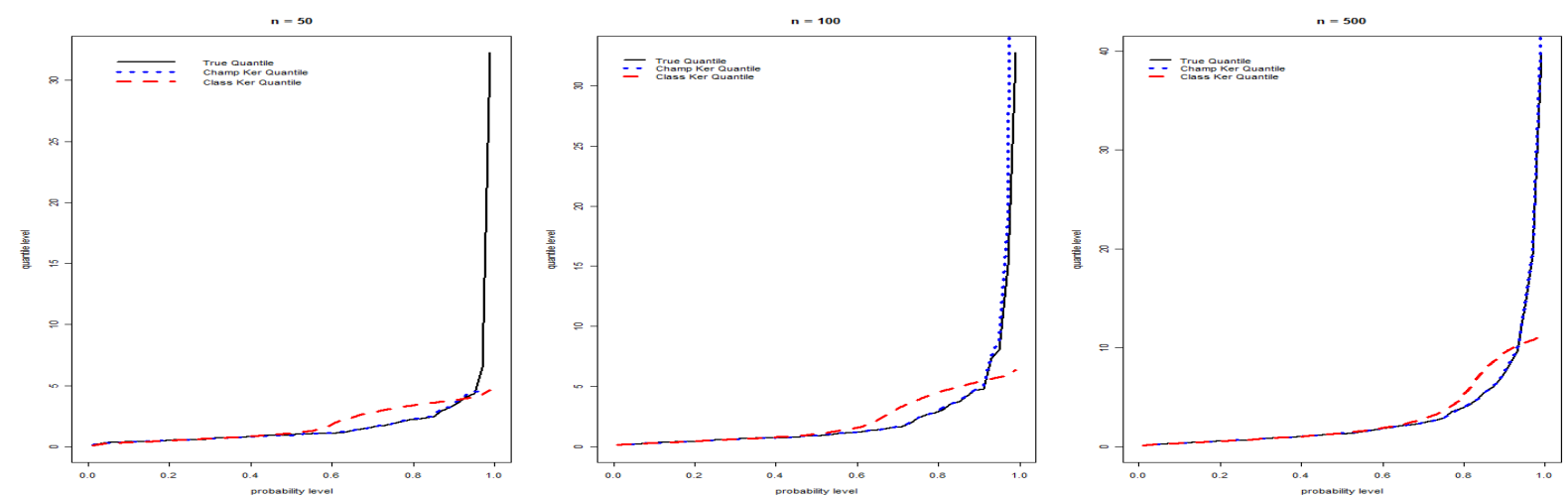

Figure 3: True quantile, classical and transformed $p^{\text {th }}$ quantile estimators : Mixtures distribution $(\rho=.3), n=50,100$ and $500, p \in(0,1)$
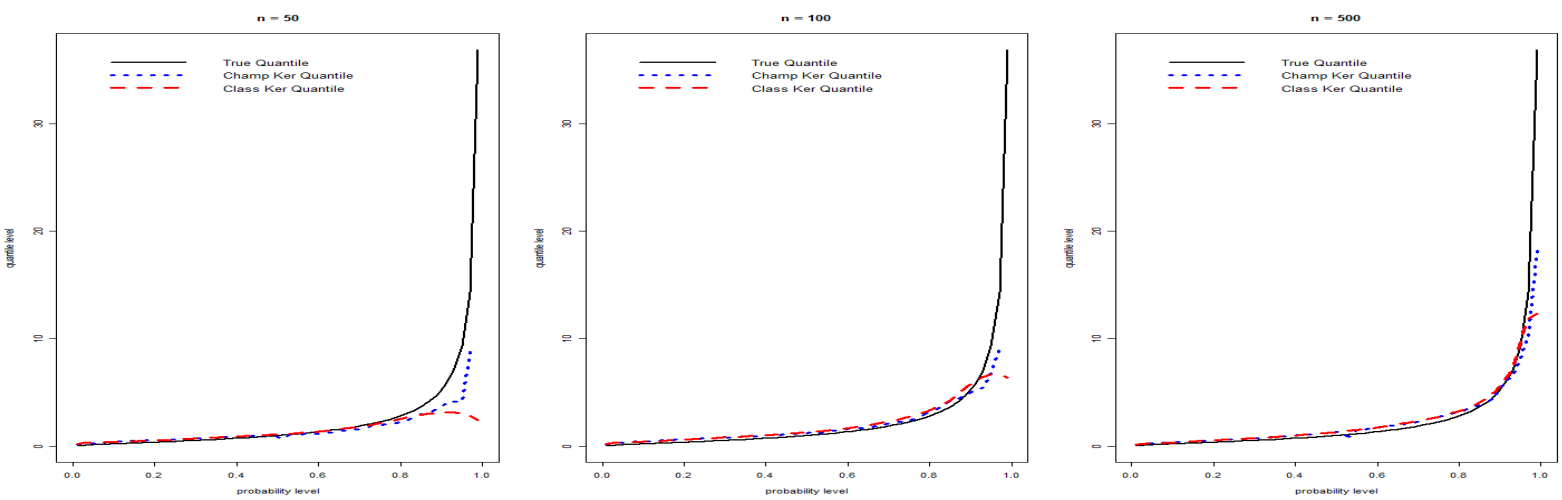

Figure 4: True quantile, classical and transformed $p^{\text {th }}$ quantile estimators : Mixtures distribution $(\rho=.7), n=50,100$ and 500, $p \in(0,1)$

Table 2. Burr distribution, 200 samples of size 200.

\begin{tabular}{llllllll}
\hline$p$ & 0.05 & 0.1 & 0.25 & 0.5 & 0.75 & 0.9 & 0.95 \\
\hline$Q(p)$ & 0.2962 & 0.3782 & 0.5368 & 0.7454 & 1.0000 & 1.2931 & 1.5143 \\
$T K Q E$ & 0.2966 & 0.3728 & 0.5345 & 0.7480 & 0.9946 & 1.2928 & 1.5150 \\
$C K Q E$ & 0.2988 & 0.3741 & 0.5345 & 0.7503 & 0.9852 & 0.5464 & 0.0367 \\
\hline
\end{tabular}


A. Sayeh, D. Yahia and A. Necir, Journal Afrika Statistika, Vol. 5, Nº12, 2010, page 288-296.

Champernowne transformation in kernel quantile estimation for heavy-tailed distributions

Table 3. Paralogistic distribution, 200 samples of size 200.

\begin{tabular}{llllllll}
\hline$p$ & 0.05 & 0.1 & 0.25 & 0.5 & 0.75 & 0.9 & 0.95 \\
\hline$Q(p)$ & 0.1075 & 0.1551 & 0.2622 & 0.4291 & 0.6667 & 0.9803 & 1.2422 \\
$T K Q E$ & 0.7983 & 0.1278 & 0.2526 & 0.4263 & 0.6705 & 0.9676 & 1.1626 \\
$C K Q E$ & 0.1088 & 0.1547 & 0.2641 & 0.4330 & 0.7024 & 0.6079 & 0.4421 \\
\hline
\end{tabular}

Table 4. Mixtures ( $r h o=0.3$ ) distribution, 200 samples of size 200.

\begin{tabular}{llllllll}
\hline$p$ & 0.05 & 0.1 & 0.25 & 0.5 & 0.75 & 0.9 & 0.95 \\
\hline$Q(p)$ & 0.0948 & 0.1611 & 0.3862 & 1.0000 & 2.6889 & 7.3807 & 14.8541 \\
$T K Q E$ & 0.2380 & 0.3391 & 0.6213 & 1.2560 & 2.7743 & 7.2812 & 15.2085 \\
$C K Q E$ & 0.2350 & 0.3380 & 0.6273 & 1.3246 & 16.4845 & 28.9263 & 21.5483 \\
\hline
\end{tabular}

Table 5. Mixtures ( $r h o=0.7)$ distribution, 200 samples of size 200.

\begin{tabular}{llllllll}
\hline$p$ & 0.05 & 0.1 & 0.25 & 0.5 & 0.75 & 0.9 & 0.95 \\
\hline$Q(p)$ & 0.1509 & 0.2277 & 0.4566 & 1.0000 & 2.2741 & 5.2216 & 9.3262 \\
$T K Q E$ & 0.2987 & 0.4200 & 0.7230 & 1.3483 & 2.5389 & 5.1070 & 8.4522 \\
$C K Q E$ & 0.3239 & 0.3981 & 0.7293 & 1.3805 & 2.6514 & 6.6738 & 29.6183 \\
\hline
\end{tabular}

Table 6. Classical and transformed pth quantile estimators and their MSE, p = .75 and 200 replications

\begin{tabular}{|c|c|c|c|c|c|c|}
\hline \multicolumn{3}{|c|}{ Distribution } & \multirow{3}{*}{$\begin{array}{l}\text { Burr } \\
\mathbf{1 . 0 0 0 0}\end{array}$} & \multirow{3}{*}{$\begin{array}{l}\text { Paralogistic } \\
\mathbf{0 . 6 6 6 7}\end{array}$} & \multicolumn{2}{|c|}{$\rho \log$ normal and $(1-\rho)$ Pareto } \\
\hline & & & & & $\rho=30 \%$ & $\rho=70 \%$ \\
\hline \multicolumn{2}{|c|}{$p=.75$} & $Q(p)$ & & & 2.6889 & 2.2741 \\
\hline \multirow[t]{4}{*}{$n=50$} & value & $T K Q E$ & 0.9623 & 0.6622 & 2.7235 & 2.6067 \\
\hline & & $C K Q E$ & 0.7963 & 0.7059 & 7.0750 & 3.0655 \\
\hline & $A M S E$ & $T K Q E$ & 0.0150 & 0.0059 & 1.0175 & 0.3999 \\
\hline & & $C K Q E$ & 0.0445 & 0.0080 & 106.46 & 1.1058 \\
\hline \multirow[t]{4}{*}{$n=100$} & value & $T K Q E$ & 0.9912 & 0.6627 & 2.8756 & 2.5885 \\
\hline & & $C K Q E$ & 0.8922 & 0.7256 & 46.800 & 2.7845 \\
\hline & $A M S E$ & $T K Q E$ & 0.0048 & 0.0029 & 0.3518 & 0.2383 \\
\hline & & $C K Q E$ & 0.0135 & 0.0069 & 30501 & 0.4163 \\
\hline \multirow[t]{4}{*}{$n=500$} & value & $T K Q E$ & 1.0027 & 0.6664 & 2.7815 & 2.5781 \\
\hline & & $C K Q E$ & 1.0479 & 0.6825 & 3.2990 & 2.6369 \\
\hline & $A M S E$ & $T K Q E$ & 0.0008 & 0.0006 & 0.0553 & 0.1151 \\
\hline & & $C K Q E$ & 0.0030 & 0.0008 & 0.4490 & 0.1522 \\
\hline
\end{tabular}

\section{Conclusion}

For heavy-tailed distributions, bias or inefficiency problems may occur in the classical kernel quantile estimation when considering high probability levels. In this paper, we have solved this incontinence by using a new approach based on the modified Champernowne distribution which behaves as the Pareto distribution. Therefore it can capture the thick-tail feature exhibited by empirical loss data. The transformation step can also be seen as a kind of variance stabilization procedure as traditionally used in statistic sampling. Our main conclusion is that the transformed kernel quantile estimator is recommended for heavy-tailed models.

Acknowledgement : The authors are grateful to an anonymous referee whose careful reading gave them the opportunity to improve the quality of the paper. 
A. Sayeh, D. Yahia and A. Necir, Journal Afrika Statistika, Vol. 5, Nº12, 2010, page 288-296.

Champernowne transformation in kernel quantile estimation for heavy-tailed distributions

Table 7. Classical and transformed pth quantile estimators and their MSE, p =.9 and 200 replications

\begin{tabular}{|c|c|c|c|c|c|c|}
\hline \multirow{2}{*}{\multicolumn{3}{|c|}{ Distribution }} & \multirow{3}{*}{$\begin{array}{l}\text { Burr } \\
\mathbf{1 . 2 9 3 1}\end{array}$} & \multirow{3}{*}{$\begin{array}{l}\text { Paralogistic } \\
\mathbf{0 . 9 8 0 3}\end{array}$} & \multicolumn{2}{|c|}{$\rho$ log normal and $(1-\rho)$ Pareto } \\
\hline & & & & & \multirow{2}{*}{$\begin{array}{l}\rho=30 \% \\
\mathbf{7 . 3 8 0 7}\end{array}$} & \multirow{2}{*}{$\begin{array}{l}\rho=70 \% \\
\mathbf{5 . 2 2 1 6}\end{array}$} \\
\hline \multicolumn{2}{|c|}{$p=.90$} & $Q(p)$ & & & & \\
\hline \multirow[t]{4}{*}{$n=50$} & value & $T K Q E$ & 1.2941 & 0.9796 & 7.8530 & 5.2474 \\
\hline & & $C K Q E$ & 0.3864 & 0.4683 & 10.668 & 9.5797 \\
\hline & $A M S E$ & $T K Q E$ & 0.0201 & 0.0277 & 15.545 & 3.2335 \\
\hline & & $C K Q E$ & 0.8230 & 0.2655 & 298.59 & 179.86 \\
\hline \multirow[t]{4}{*}{$n=100$} & value & $T K Q E$ & 1.2985 & 0.9819 & 7.3484 & 5.1982 \\
\hline & & $C K Q E$ & 0.4690 & 0.5341 & 12.540 & 11.3100 \\
\hline & $A M S E$ & $T K Q E$ & 0.0084 & 0.0113 & 5.3956 & 1.5319 \\
\hline & & $C K Q E$ & 0.6798 & 0.2012 & 352.99 & 324.23 \\
\hline \multirow[t]{4}{*}{$n=500$} & value & $T K Q E$ & 1.2996 & 0.9773 & 6.9729 & 4.9967 \\
\hline & & $C K Q E$ & 0.6399 & 0.7219 & 22.028 & 5.3940 \\
\hline & $A M S E$ & $T K Q E$ & 0.0020 & 0.0021 & 1.0575 & 0.2473 \\
\hline & & $C K Q E$ & 0.4269 & 0.0679 & 698.79 & 0.2868 \\
\hline
\end{tabular}

Table 8. Classical and transformed pth quantile estimators and their MSE, p $=.95$ and 200 replications

\begin{tabular}{|c|c|c|c|c|c|c|}
\hline \multicolumn{3}{|c|}{ Distribution } & \multirow{3}{*}{$\begin{array}{l}\text { Burr } \\
\mathbf{1 . 5 1 4 3}\end{array}$} & \multirow{3}{*}{$\begin{array}{r}\text { Paralogistic } \\
\mathbf{1 . 2 4 2 2}\end{array}$} & \multicolumn{2}{|c|}{$\rho \log$ normal and $(1-\rho)$ Pareto } \\
\hline & & & & & $\rho=30 \%$ & $\rho=70 \%$ \\
\hline \multicolumn{2}{|c|}{$p=.95$} & $Q(p)$ & & & 14.8541 & 9.3262 \\
\hline \multirow[t]{4}{*}{$n=50$} & value & $T K Q E$ & 1.5506 & 1.0945 & 16.6389 & 9.0187 \\
\hline & & $C K Q E$ & 0.0232 & 0.3396 & 12.2710 & 12.0748 \\
\hline & $A M S E$ & $T K Q E$ & 0.0443 & 0.0751 & 165.422 & 19.7341 \\
\hline & & $C K Q E$ & 2.2232 & 0.8165 & 1025.83 & 466.674 \\
\hline \multirow[t]{4}{*}{$n=100$} & value & $T K Q E$ & 1.5332 & 1.1352 & 14.8011 & 8.6076 \\
\hline & & $C K Q E$ & 0.0291 & 0.3889 & 16.0566 & 17.5289 \\
\hline & $A M S E$ & $T K Q E$ & 0.0211 & 0.0702 & 42.2056 & 4.8286 \\
\hline & & $C K Q E$ & 2.2057 & 0.7294 & 1129.14 & 669.036 \\
\hline \multirow[t]{4}{*}{$n=500$} & value & $T K Q E$ & 1.5181 & 1.1740 & 14.4662 & 8.1453 \\
\hline & & $C K Q E$ & 0.0498 & 0.5174 & 28.3102 & 27.2626 \\
\hline & $A M S E$ & $T K Q E$ & 0.0038 & 0.0468 & 9.4011 & 2.8212 \\
\hline & & $C K Q E$ & 2.1447 & 0.5259 & 2123.63 & 9055.37 \\
\hline
\end{tabular}

\section{References}

[1] Azzalini, A., 1981. A note on the estimation of a distribution function and quantiles by a kernel method. Biometrika 68, 326-328.

[2] Buch-Larsen, T., Guillén, M., Neilson J.P. and Bolancé, C. 2005. Kernel density estimation for heavy-tailed distributions using the Champernowne transformation. Statistics 39, 503-518.

[3] Bolancé, C., Guillén, M. and Neilson J.P., 2008. Inverse beta transformation in kernel density estimation. Statist. Es Prob. Letters 78, 1757-1764.

[4] Champernowne, D.G., 1936. The Oxford meeting, September 25-29, by Brown P. Econometrica 5, 361-383.

[5] Champernowne, D.G., 1952. The graduation of income distributions. Econometrica 20, 591-615.

[6] Charpentier, A. and Oulidi, A., 2010. Beta kernel quantile estimators of heavy-tailed loss distributions. Stat. Comput. 20, $35-55$.

[7] Chen,S.X. and Tang, C.Y., 2005. Nonparametric inference of values at risk for dependent financial returns. J. Econ. Finance 3, 227-255.

[8] Denuit, M., Dhaene, J., Goovaerts, M.J. and Kaas, R., 2005. Actuarial Theory for Dependent Risk: Measures, Orders and Models. Wiley, New York.

[9] Devroye, L. and Györfi, L., 1985. Nonparametric Density Estimation: The L1 View. Wiley, New York.

[10] Falk, M., 1984. Relative deficiency of the kernel type estimators of quantiles. Ann. Statist. 12, 216-268.

[11] Harrell, F.E. and Davis, C.E., 1982. A new distribution free quantile estimators. Biometrika 69, 635-640.

[12] Johnson, N.L., Jotz, S. and Balakrishnan, N., 1995. Continuous Univariate Distributions. Vol.1. John Wiley \& Sons, Inc., New York. 
[13] Jones, M.C., 1990. Variable kernel density estimation and variable kernel density estimation. Aust. J. Statist. 32, 361-371.

[14] Lehmann, E.L., 1991. Theory of Point Estimation. Wadsworth and Brooks/Cole, Belmont.

[15] Parzen, E., 1979. Nonparametric statistical data modeling (with discussion). J. Amer. Statist. Assoc. 74, $105-131$.

[16] Ralescu, S.S. and Sun, S., 1993. Necessary and sufficient conditions for the asymptotic normality of perturbed sample quantiles. J. Statist. Plan. and Inf. 35, 55-64.

[17] Reiss, R.D., 1981. Nonparametric estimation of smooth distribution functions. Scand. J. Statist. 9, 65-78.

[18] Serfling, R.J., 1980. Approximation theorems of mathematical statistics. John Wiley, New York.

[19] Sheather, S.J. and Marron, J.S., 1990. Kernel quantile estimators. J. Amer. Statist. Assoc. 85, 410-416.

[20] Silverman, B.W., 1986. Density Estimation for Statistics and Data Analysis. Chapman \& Hall, London.

[21] Wand, M.P. and Jones, M.C., 1995. Kernel Smoothing. Chapman \& Hall, London.

[22] Yang, S.S., 1985. A smooth nonparametric estimator of a quantile function. J. Amer. Statist. Assoc. 80, 1004-1011. 